\title{
Ultra-Wideband Metamaterial-Loaded Microstrip Array Antennas using Fibonacci \& Fractal Geometric Patterns, Design and Modelling
}

\author{
Besharat Rezaei Shookooh, Alireza Monajati, and Hamid Khodabakhshi
}

\begin{abstract}
In this study; theory, design and modeling of a new topology of ultra-wideband (UWB) metamaterial (MTM) loaded microstrip array antenna using Fibonacci \& fractal geometric patterns are analyzed. This antenna is made basically from many monopole elements which are loaded by complementary metamaterial transmission line (CMTL) unit cells. The distributed CMTL element, contains a Koch-shaped expanded complementary single split ring resonator (CSRR) pair. At first step, the UWB microstrip array antenna designed with two CMTL-loaded monopole element. So, using an iterative method based on the Fibonacci and fractal geometry patterns, the array antenna is expanded. As the order of iteration increases, the impedance bandwidth of the proposed array antennas improve more, and the radar cross section (RCS) decreases. the impedance bandwidth of the proposed third-order Fibonacci and fractal CMTL-loaded array antennas are $250 \mathrm{MHz}$ and $539 \mathrm{MHz}$ more than the twoelement CMTL-loaded array antenna, respectively. Finally, this paper presents an improved method to extract the parameters of an equivalent circuit model of the proposed MTM-loaded array antenna. Verification of the equivalent model have been validated utilizing ADS software. The obtained model, in addition to numerically efficient in comparison with the full wave analysis utilizing the moment method, gives a good physical insight to the mutual coupling mechanism of the array antenna.
\end{abstract}

Index Terms - modeling, increasing bandwidth, Metamaterial, Fibonacci geometry, Fractal geometry, radar cross section, equivalent circuit.

\section{INTRODUCTION}

Much effort has been made in recent years to improve the operation of UWB microstrip antennas in view of rapid advances in wireless communication [1], [2]. Two important specifications of a UWB microstrip antenna consist of a stable gain and impedance matching at $3.1 \sim 10.6 \mathrm{GHz}$ frequency bandwidth [3]. Various techniques have been proposed to meet these specifications, such as the use of a dielectric substrate with high permittivity, Defected Microstrip Structure (DMS), Metamaterial (MTM) loading, Defected Ground Structure (DGS) in the ground plane, or a mixture of all of them [4]-[6]. The need for antennas with

Published on October 30, 2020.

Besharat Rezaei Shookooh, Yadegar-e-Imam Khomeini (RAH) Shahre Rey Branch, Islamic Azad University, Tehran, Iran.

e-mail: te.besharat ${ }^{@}$ gmail.com)

Alireza Monajati, Yadegar-e-Imam Khomeini (RAH) Shahre Rey Branch, Islamic Azad University, Tehran, Iran.

((corresponding e-mail: alireza.monajati@yahoo.com)

Hamid Khodabakhshi, Yadegar-e-Imam Khomeini (RAH) Shahre Rey Branch, Islamic Azad University, Tehran, Iran

(e-mail: Khodabakhshi.hamid@gmail.com) small dimensions, low price, and wide bandwidth is increasing [7], [8]; However, while many microstrip antenna designs offer these features, the present paper suggests that a low-profile printed array antenna may be good due to its ease of construction and overall robust structure.

Synthetic electromagnetic MTMs have properties that are not commonly found in nature [9]-[12]. In order to create an MTM, there are two methods: non-resonant and resonant [13]. Resonant method generate an MTM by inducing electric dipole and magnetic moments in order to achieve negative permittivity and permeability, respectively. Of course, MTMs designed using this method have narrow bandwidth because of high quality factor of their resonant structure [3]. This study presents a UWB MTM-loaded microstrip array antenna that is composed of MTM radiation unit cells printed in a planar structure. Also, MTM unit cells are designed by resonance method.

B. B. Mandelbort introduced Fractal geometry at 1975 . Then, researchers utilized Fractal geometry within the design of various antenna structures [14]. Sierpinski, Minkowski, and Koch geometries are the famous fractal shapes that are utilized as multiband and wideband antennas according to their self-similarity specification. Fractal tree is another desirable fractal structure that is utilized in antenna topologies [15], [16]. In this paper, design of a fractal MTM tree microstrip array antenna was proposed and the effect of the geometry and number of the branches of the proposed structure at antenna specifications could be investigated [14], [17].

Fibonacci popularized the Hindu-Arabic numeral system in the Western World primarily through his composition in 1202 of "Liber Abaci" (Book of Calculation). He also introduced Europe to the sequence of Fibonacci numbers, which he used as an example in "Liber Abaci" [14], [18]. In the Fibonacci sequence, each number is the sum of the previous two numbers (in mathematical terms, $F_{n}=F_{n-1}+F_{n}$ 2), so the Fibonacci sequence could be: 1, 1, 2, 3, 5, 8, 13, etc. The geometric description of the Fibonacci sequence is very common in nature such as the petals of several flowers, artichokes, mature sunflowers, pine cones, pineapples, and the spiral arrangements of plant leaves [14], [18]. The branch geometry of the proposed MTM-loaded microstrip array antenna was designed due to the Fibonacci sequence and extends from the top of the antenna to its base. Fig. 1 shows some samples of fractal and Fibonacci geometries exist in nature. 


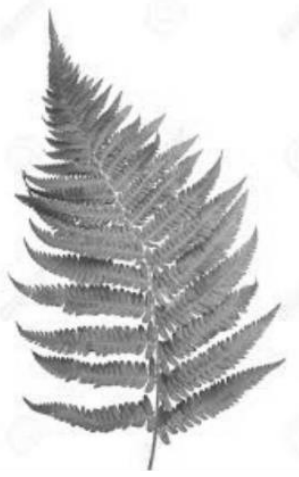

(a)

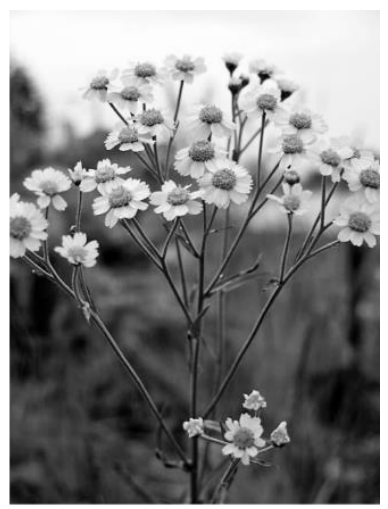

(b)
Fig. 1. Samples of fractal and Fibonacci geometries exist in nature, respectively: (a) Fern (b) Sneezewort.

Providing the equivalent circuit model of an antenna is very important theoretically and practically. An accurate equivalent model, which is extracted with respect to the physical parameters and geometry of the antenna, can give useful insights into the design and performance of the antenna [19]. Usually, the array factor is computed with elements independent for each other. While, the mutual coupling between elements can affect the array's performances. So, in order to obtain accurate equivalent model of an array, the mutual coupling between elements should be consider [20]. In our research we propose a technique to calculate the equivalent circuit model parameters of the array. In the proposed model, we consider the mutual coupling in the case of a planar array. In order to build our model, we began by analyzing the CMTL-loaded monopole element, then we used two elements, finally we generalized the technique in order to build an electrical model of proposed array. The introduced model is very important because in one hand it helps reducing the time of simulation and in the other hand, the proposed model can help us to display the design of an array taking in consideration all kind of coupling between different elements [20]. In order to evaluate our model, we compared the results given by the electrical model ADS simulator and those obtained by using HFSS simulator which is based on FEM method.

This paper presents a novel design and model of UWB MTM-loaded microstrip array antennas based on the Fibonacci \& fractal geometric patterns. The radiating elements of the antenna are made from MTM-inspired microstrip-fed monopoles placed along one axis. Section 2 presents analyzes of the CMTL element. Section 3 presents designing of the UWB microstrip array antenna with two radiation CMTL-loaded elements. In section 4, theory and design of the proposed UWB MTM-loaded microstrip array antennas based on the Fibonacci \& fractal geometric patterns are investigated. Section 5 discusses approaches for representing the proposed first-iteration fractal \& Fibonacci MTM-loaded array antennas by equivalent circuit model. Section 6 concludes the whole paper.

\section{THE CMTL ELEMENT}

Fig. 2 shows the layout of the CMTL element. As can be seen, the CMTL element consists of an inner Koch-shaped extended complementary single split ring resonator pair (KECSSRRP) slot (depicted in white) etched on the square patch of a conductor strip (depicted in gray) [7]. The KECSSRRP evolves from the complementary single split ring resonator pair (CSSRRP) by expanding each end point of the CSSRRP with an inner smaller slot made of three second-order Koch curves and one first-order curve. The dimensions of the CMTL element shown in Fig. 2 are as follows (in millimeter: $\mathrm{mm}$ ): $\mathrm{a}=1.325, \mathrm{~b}=2.575, \mathrm{c}=0.075$, $\mathrm{g}=0.075$ [7].

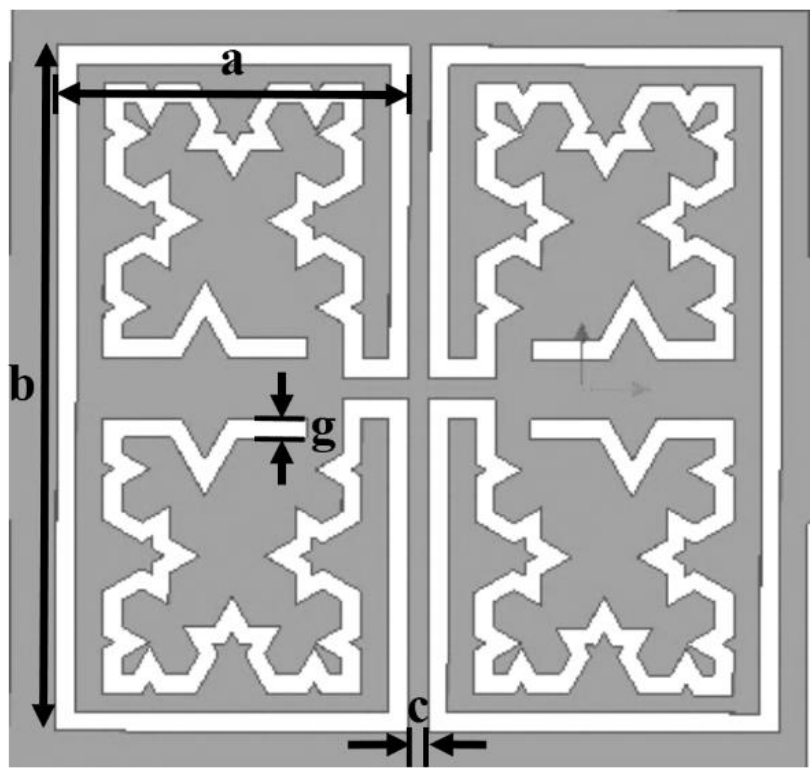

Fig. 2. Layout of the CMTL element [7].

Permittivity $(\varepsilon)$ and permeability $(\mu)$ can describe electrically materials in the frequency domain and determine the response of the material to electromagnetic radiation. This study utilized a retrieval method using the $\mathrm{S}$ parameters in order to obtain the curves for the permittivity and permeability of the MTM unit cell. HFSS simulator which is based on FEM, utilized in order to extract the S parameters of MTM unit cell [1], [21]. In order to simulate the periodic boundary conditions, as shown in Fig. 3(a) and 3(b), The composition of perfect electric conductor (PEC) and perfect magnetic conductor (PMC) conditions utilized due to the symmetry inherited by the MTM. After determination of the periodic boundary conditions, as shown in Fig. 3(c), in order to simulate the incident wave that propagated from the top to bottom of the unit cell, the waveguide excitation ports were used. The S-parameter curves of the MTM element that extracted by HFSS are shown in Fig. 4.

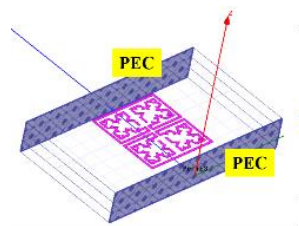

(a)

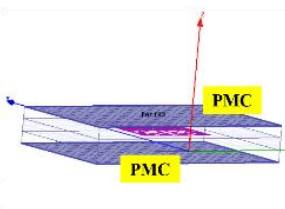

(b)

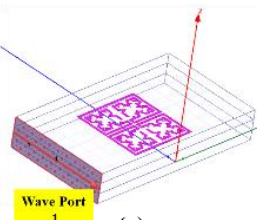

(c)
Fig. 3. The simulation of the CMTL element with periodic boundary condition: (a) PEC planes (b) PMC planes (c) Wave ports (Port 1). 


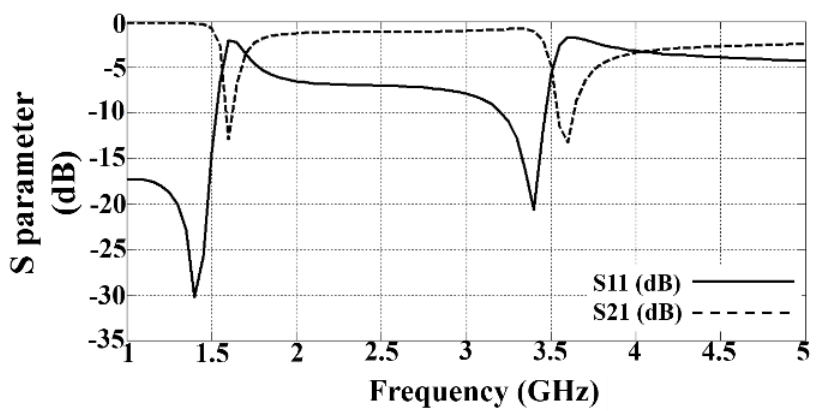

Fig. 4. The S-parameter curves of the CMTL element.

By the following expressions, Permittivity $(\varepsilon)$ and Permeability $(\mu)$ are related to refractive index and the impedance [21]:

$$
\begin{aligned}
& z= \pm \sqrt{\frac{\left(1+S_{11}\right)^{2}-S_{21}{ }^{2}}{\left(1-S_{11}\right)^{2}-S_{21}{ }^{2}}} \\
& e^{j n k_{0} d}=\frac{S_{21}}{1-S_{11} \frac{z-1}{Z+1}}, \\
& n=\frac{1}{k_{0} d}\left[\left\{\operatorname{Im}\left[\ln \left(e^{j n k_{0} d}\right)\right]+2 m \pi\right\}-j\left[\operatorname{Re}\left[\ln \left(e^{j n k_{0} d}\right)\right]\right]\right] \\
& \varepsilon=\frac{n}{z} \\
& \mu=n z
\end{aligned}
$$

where $\mathrm{n}$ is the refractive index, $\mathrm{d}$ is the maximum length of the unit element, $\mathrm{m}$ is the branch due to the periodicity of the sinusoidal function, $\mathrm{k}_{0}$ is the wavenumber and $\mathrm{z}$ is the input impedance.

Finally, the complex permittivity and permeability curves were obtained using a MATLAB script which used the presented formulations [1], [18] (Fig. 5(a) and (b)). Also, the refractive index and the impedance of the MTM unit cell are shown in Figs. 5(c) and 5(d). As shown in Figure 5, the MTM unit cell has a negative refractive index around the resonant frequency.

\section{THE UWB MTM-LOADED MICROSTRIP ARRAY ANTENNA}

As show in Fig. 6a, a microstrip monopole patch antenna that was loaded by the MTM unit cell, utilized to design a two-element microstrip array antenna. A dual-shaped feed structure utilized in the array antenna design to excitation of the MTM-loaded monopoles and promote an even field distribution. The dimension of the top layer of the array antenna is $21 \times 21 \mathrm{~mm}^{2}$. Other dimensions of the array antenna structure as shown in Fig. 6(a), are as follows: (in millimeter: $\mathrm{mm})$ : $\mathrm{W}_{1}=10.5, \mathrm{~W}_{2}=2, \mathrm{~W}_{3}=3.5, \mathrm{~W}_{4}=2.75$, $\mathrm{W}_{5}=11.2, \mathrm{~W}_{6}=1, \mathrm{~W}_{7}=2.85, \mathrm{~W}_{8}=3$, and $\mathrm{d}=9.2$.

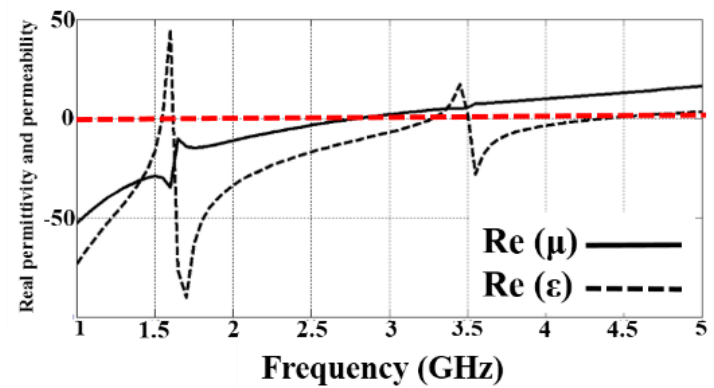

(a)

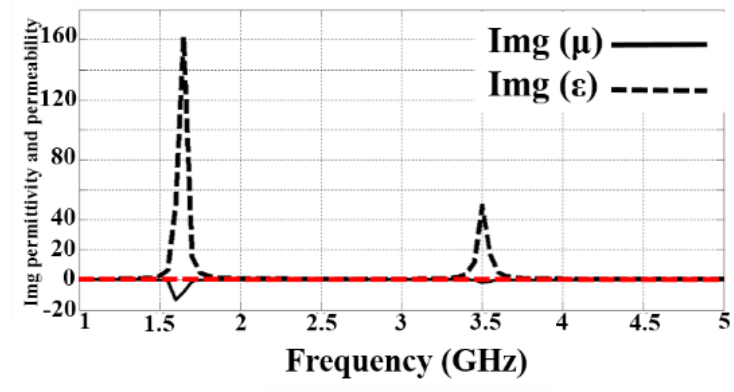

(b)

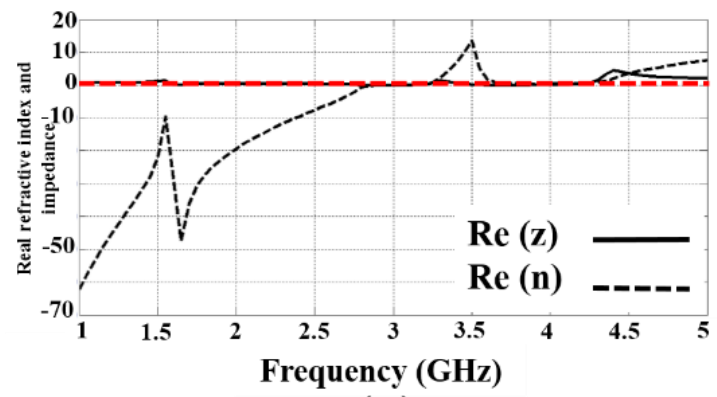

(c)

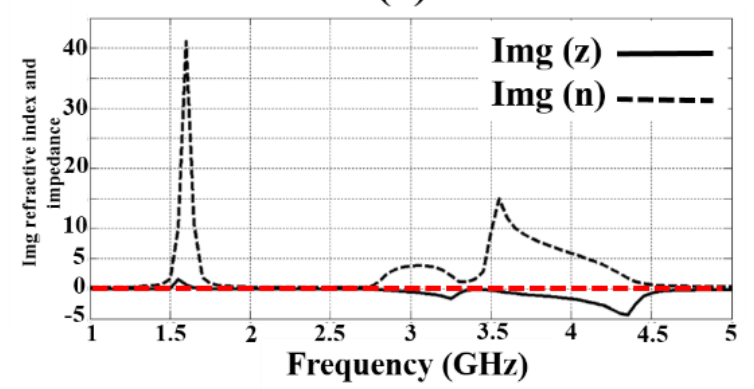

(d)

Fig. 5. (a) The real curves and (b) The imaginary curves of the permeability and permittivity, (c) The real curves and (d) The imaginary curves of the impedance and refractive index.

The impedance bandwidth of the Structures with low energy storage, are wider due to their low Q factor. So, in order to increase the bandwidth of the array antenna and improve matching, a partial ground plane with $\mathrm{W}_{\mathrm{GP}}=7.5$ $\mathrm{mm}$ was utilized. Furthermore, a slot with dimension $5 \times 3$ $\mathrm{mm}^{2}\left(\mathrm{~L}_{\mathrm{s}} \times \mathrm{W}_{\mathrm{s}}\right)$ was introduced to the partial ground plane, in order to gain more improvements. In order to obtain the wideband matching, the $\mathrm{L}_{S}$ and $\mathrm{W}_{\mathrm{S}}$ parameters were optimized. The layout of the bottom layer of the array antenna is shown in Fig. 6 (b). 


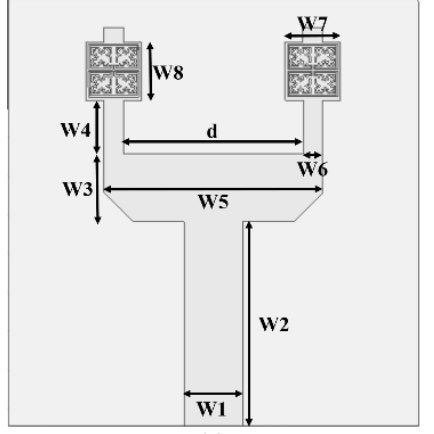

(a)

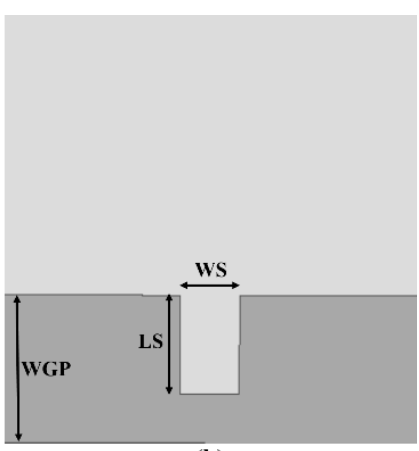

(b)
Fig. 6. The layout of the MTM-loaded two-element microstrip array antenna: (a) Top layer (b) bottom layer.

HFSS Software was used to analyze the UWB MTMloaded two-element array antenna schematic. The substrate is FR4 with thickness $1.6 \mathrm{~mm}$, loss tangent of about 0.025 , and a dielectric constant $\varepsilon_{\mathrm{r}}=4.3$. The simulation results of the impedance bandwidth of the MTM-loaded two-element array antenna is shown in Fig. 7. It can be seen that the -10 $\mathrm{dB}$ impedance bandwidth is 3.36 9.48 GHz. However, the simulated return loss is a little above than $-10 \mathrm{~dB}$ at frequency around $6.2 \mathrm{GHz}$.

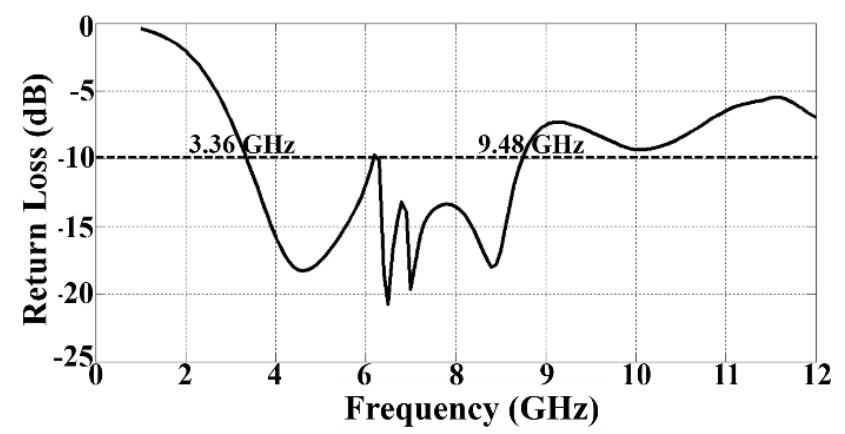

Fig. 7. HFSS simulation results of the return loss of the UWB MTM-loaded two-element array antenna.

\section{THE PROPOSED UWB MTM-LOADED MICROSTRIP ARRAY ANTENNA}

In this paper, a method to design a new family of UWBMTM-loaded array antennas using Fibonacci and fractal geometric patterns was proposed. In the related papers, it was found that the properties of fractal geometry affect the electromagnetic behavior of the antenna. Fractal tree geometry with uniform branch length ratios, utilized in microstrip antenna design in many recent studies. As shown in Fig. 8 (a), the branches of the fractal tree expand according to the sequence $1,2,4,8,16,32$, etc. and the branch length ratio is $1 / 2$.

The Fibonacci tree geometry is shown in Fig. 8(b), which the branches expand according to the Fibonacci sequence, it can be seen that the complexity in comparison with the fractal tree geometry decreased significantly. Also, due to determining the number of branches based on the Fibonacci sequence, branch length ratios is non-uniform. The geometry of Dream tree is shown in Fig. 8(c), which the branches expand according to the modified Fibonacci sequence: $1,2,3,5,8,13$, etc. This geometry is based on the growth algorithm of the rabbit population that Fibonacci found.
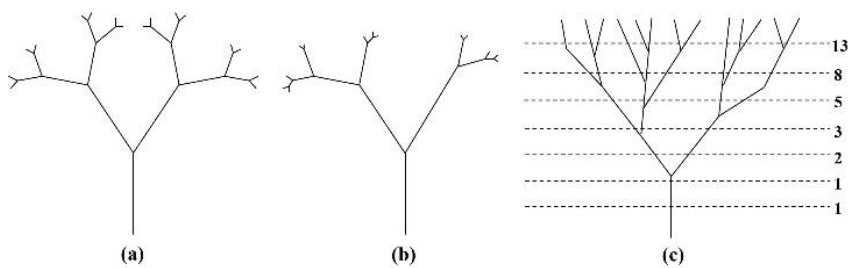

Fig. 8. The geometry of the proposed microstrip array antenna: (a) Fractal tree (b) Fibonacci tree (c) Dream tree.

The proposed Fractal UWB MTM-loaded array is shown in Fig. 9, which was resulted of applying an iterative method to the MTM-loaded two-element array. At the first step, each radiation element split into two similar element with a $1 / 2$ ratio. Then, this iterative method continues in order to obtain second- and third-order structures. As shown in Fig. 9, the branches expanded due to the sequence: $1,2,4,8$, etc. and the branch dimension ratios are uniform, which are 1/2.

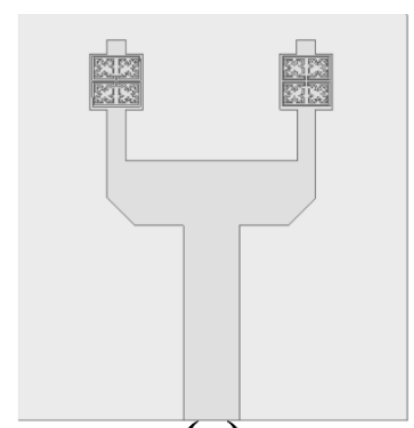

(a)

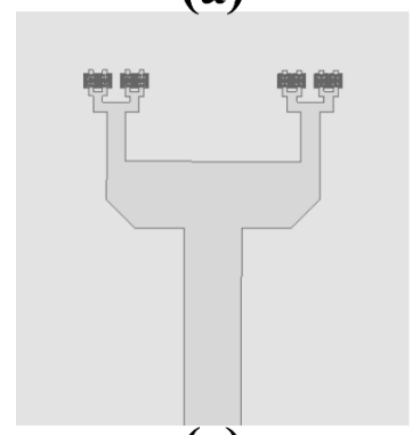

(c)
Fig. 9. The layout of the proposed fractal UWB MTM-loaded array antenna: (a) First-order (b) Second-order (c) Third-order.

As shown in Fig. 10, the particular proposed topology of the microstrip array antenna designed based on Fibonacci sequence. The method of design is almost similar as the previous fractal proposed array. As shown in Fig. 10(b), at the first step, one radiation branch of the MTM-loaded two element array split to two branches with $1 / 2$ ratio, and the other radiation branch remains in position without change. The second- and third-order structures of the proposed Fibonacci UWB MTM-loaded array was obtained by applying this iterative method to previous order structure, as shown in Fig. 10(c). It can be seen that the branches of the proposed array expanded based on the Fibonacci sequence: $1,2,3,5,8,13$, etc. and the branch dimension ratios are non-uniform due to non-uniform geometry of the dream tree. 


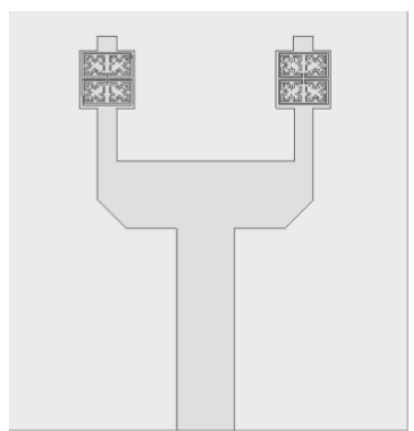

(a)

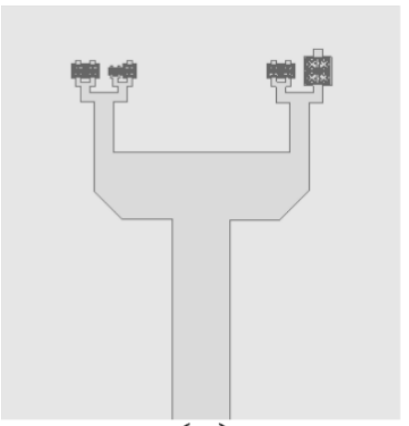

(c)

Fig. 10. The proposed structure of the Fibonacci UWB MTM-loaded microstrip array antenna: (a) First-order (b) Second-order (c) Third-order.

In order to compare the proposed array antennas with the two-element MTM-loaded array, HFSS simulator was utilized to analyze the structures with the same substrate. The simulated return loss results and metal areas of the array antennas are listed in Table 1. As shown in this table, the impedance bandwidth of the proposed Third-order Fibonacci and fractal UWB MTM-loaded array antennas are $250 \mathrm{MHz}$ and $539 \mathrm{MHz}$ greater than two-element MTMloaded array, respectively. The MTM-loaded radiation elements of the proposed array antennas are more in comparison with Two-element array and are so closer to each other. Therefore, the near fields of the proposed array affected more due to mutual coupling between the MTMloaded radiation elements and the input impedance which is related to the near and far fields of the antenna can lead to an improvement. Also, the improvement of the proposed array antenna bandwidth can be explained from another point of view. As shown in Table 1, the metal area of the proposed array antennas decrease as the order of the structure increases. Therefore, the capacitance of the proposed array antenna patch decreased due to metal area reduction. The reduction of the capacitance leads to quality factor reduction and thus the impedance bandwidth improves. Also, according to the metal area reduction of the proposed Fibonacci and fractal array antennas, the radar cross section (RCS) decreased in comparison with the twoelement array antenna. The return loss simulation results of the two-element and the proposed array antennas are shown in Fig. 11 in order to better comparison.
TABLE 1: THE SIMULATION RESULTS OF THE FIBONACCI \& FRACTAL UWB MTM-LOADED MICROSTRIP ARRAY ANTENNAS IN COMPARISON WITH TWO-

\begin{tabular}{|c|c|c|c|c|c|}
\hline Ante & a type & $\begin{array}{c}\text { JT MTM-LO } \\
\text { Bandwidth } \\
(\mathrm{GHz})\end{array}$ & $\begin{array}{c}\text { ED ARR } \\
\text { Metal } \\
\text { Area } \\
\left(\mathrm{mm}^{2}\right) \\
\end{array}$ & $\begin{array}{l}\text { ANTENNA } \\
\text { Bandwidth } \\
\text { enhanceme } \\
\text { nt }(\mathrm{MHz})\end{array}$ & $\begin{array}{c}\begin{array}{c}\text { Metal area } \\
\text { reduction } \\
\left(\mathrm{mm}^{2}\right)\end{array} \\
\end{array}$ \\
\hline $\begin{array}{l}\text { Two-ele } \\
\text { loaded a }\end{array}$ & $\begin{array}{l}\text { nt MTM- } \\
\text { y antenna }\end{array}$ & $\begin{array}{c}3.36 \sim 9.48 \\
(6.12)\end{array}$ & $\begin{array}{c}176.50 \\
4137\end{array}$ & - & - \\
\hline $\begin{array}{l}\text { The } \\
\text { fractal }\end{array}$ & Order 1 & $\begin{array}{c}3.52 \sim 9.67 \\
(6.15)\end{array}$ & $\begin{array}{c}169.35 \\
0714\end{array}$ & 30 & $\begin{array}{c}7.153423 \\
(4.05 \%)\end{array}$ \\
\hline $\begin{array}{l}\text { UWB } \\
\text { MTM- }\end{array}$ & Order 2 & $\begin{array}{l}3.65 \sim 10.1 \\
63(6.513)\end{array}$ & $\begin{array}{c}165.38 \\
3002\end{array}$ & 393 & $\begin{array}{c}11.121135 \\
(6.3 \%)\end{array}$ \\
\hline $\begin{array}{c}\text { loaded } \\
\text { array } \\
\text { antenna }\end{array}$ & Order 3 & $\begin{array}{c}3.737 \sim 10 . \\
396 \\
(6.659)\end{array}$ & $\begin{array}{c}163.33 \\
7397\end{array}$ & 539 & $\begin{array}{c}13.16674 \\
(7.46 \%)\end{array}$ \\
\hline $\begin{array}{c}\text { The } \\
\text { Fibonac } \\
\text { ci }\end{array}$ & Order 1 & $\begin{array}{c}3.43 \sim 9.57 \\
4(6.144)\end{array}$ & $\begin{array}{c}172.92 \\
7425\end{array}$ & 24 & $\begin{array}{c}3.576712 \\
(2.02 \%)\end{array}$ \\
\hline $\begin{array}{l}\text { UWB } \\
\text { MTM- }\end{array}$ & Order 2 & $\begin{array}{c}3.56 \sim 9.75 \\
6(6.196)\end{array}$ & $\begin{array}{c}168.35 \\
8786\end{array}$ & 76 & $\begin{array}{c}8.145351 \\
(4.61 \%)\end{array}$ \\
\hline $\begin{array}{c}\text { loaded } \\
\text { array } \\
\text { antenna }\end{array}$ & Order 3 & $\begin{array}{c}3.63 \sim 10 \\
(6.37)\end{array}$ & $\begin{array}{c}166.11 \\
9229\end{array}$ & 250 & $\begin{array}{c}10.384908 \\
(5.88 \%)\end{array}$ \\
\hline
\end{tabular}

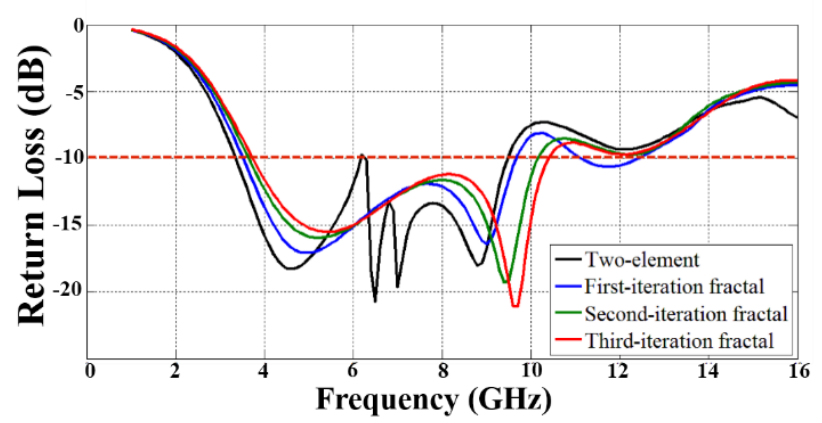

(a)

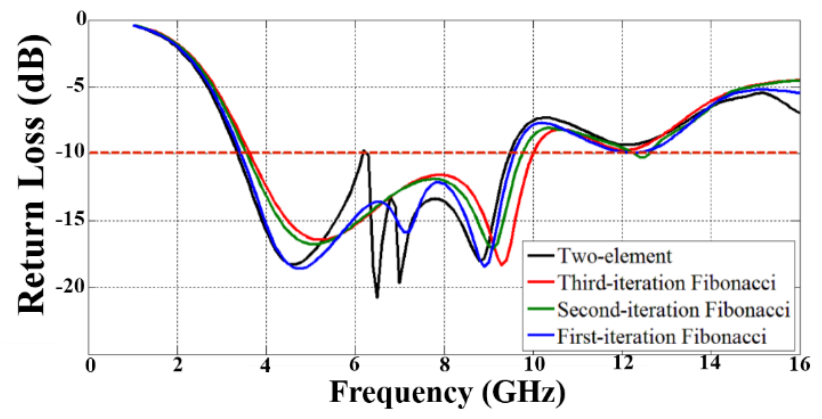

(b)

Fig. 11. The return loss simulation results of (a) the proposed fractal, (b) Fibonacci UWB MTM-loaded array antennas.

The two-element UWB MTM-loaded array antenna (Antenna 1), the proposed first-order fractal UWB MTMloaded array antenna (Antenna 2), and the proposed firstorder Fibonacci UWB MTM-loaded array antenna (Antenna 3) were analyzed by HFSS simulator at $7.3 \mathrm{GHz}$ and the comparison of their simulated $\mathrm{H}$-plane and E-plane radiation patterns are shown in Fig. 12. It can be seen that the radiation patterns of the all three antennas are similar. Antennas 2 and 3 had more radiation elements, although the radiation elements added at a higher order are smaller and fed by suitable phase and amplitude feed. Thus, the shape of the radiation patterns did not change and only resulted a little improvement in directivity. Also, the gain of the Antennas 2 and 3 increased a little in comparison with Antenna 1 due to the more number of MTM-loaded radiation elements. 


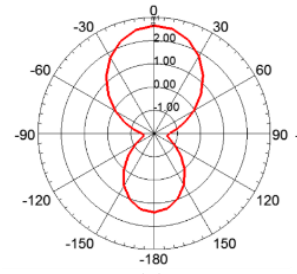

(a)

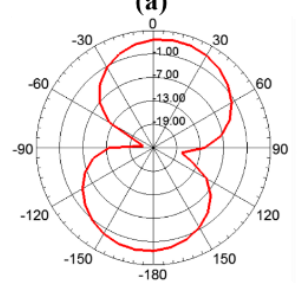

(d)

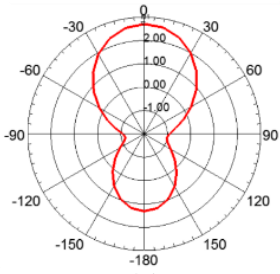

(b)

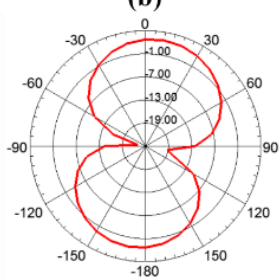

(e)

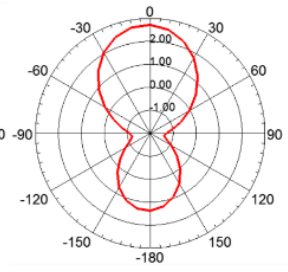

(c)

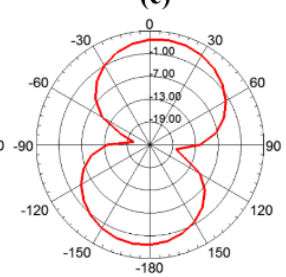

(f)
Fig. 12. HFSS Simulated H \& E-plane radiation patterns at $7.3 \mathrm{GHz}$ : (a) Eplane and (d) H-plane of the Antenna 1, (b) E-plane and (e) H-plane of the Antenna 2, (c) E-plane and (f) H-plane of the Antenna 3.

The simulated gain versus frequency curves of Antennas 1, 2 and 3 are shown in Fig. 13. As Shown, the maximum gains of Antenna 1, 2, and 3 are 2.69, 2.78, $2.73 \mathrm{dBi}$, respectively. It can be seen that the gain is maximum at frequency about $7.3 \mathrm{GHz}$ for all the antennas.

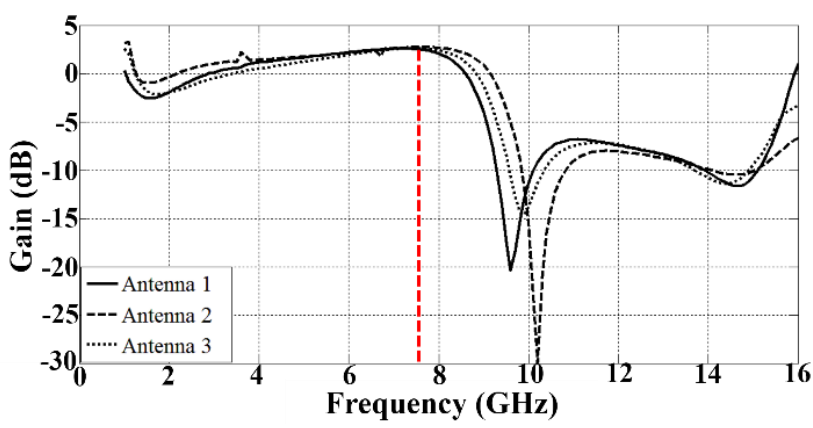

Fig. 13. The simulated Gain versus frequency curves of the Antennas 1, 2, and 3 .

In order to obtain the optimum distance between branches (d parameter), a parametric study was performed on $\mathrm{d}$ parameter of Antenna 3 (parametric sweep: $d=4 \sim 13 \mathrm{~mm}$ ). The parametric simulated return loss results of the Antenna 3 are sown in Fig14. At first glance, the red curve $(\mathrm{d}=7 \mathrm{~mm})$ seems to be optimum but around frequency $8 \mathrm{GHz}$, the return loss is slightly less than $-10 \mathrm{~dB}$. Therefore, the yellow curve $(\mathrm{d}=10 \mathrm{~mm})$ was chosen as the optimum distance according to safe and wide bandwidth in comparison with all the distances.

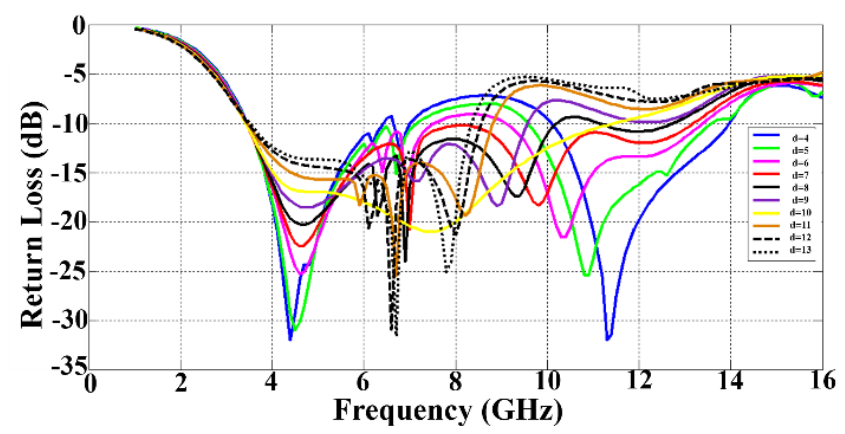

Fig. 14. The parametric simulated return loss results of Antenna 3, based on the parametric sweep of the distance between branches (d)
The simulated RCS results of the Antennas 1, 2, 3 are shown in Fig. 15. As expected, due to the reduction of metal areas, Antennas 2 and 3 have lower RCS of about 1 1.2 dB.

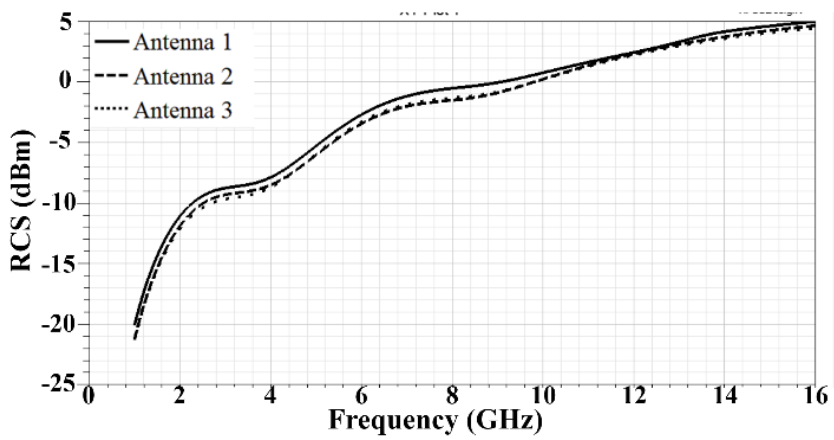

Fig. 15. The simulated RCS results of The Antennas 1, 2, and 3.

\section{THE APPROACHES TO GET EQUIVALENT CIRCUIT OF THE PROPOSED ARRAY ANTENNA}

The antenna can be represented by an equivalent circuit of several lumped elements. The input impedances of the antennas which are linear passive elements in general, can be described by Foster canonical forms, as shown in Fig. 16, which consider no ohmic loss. Fig. 16 shows The Foster canonical forms of the equivalent circuit for electric and magnetic antennas which behave as an open-circuit and short-circuit at DC input signal, respectively [19].

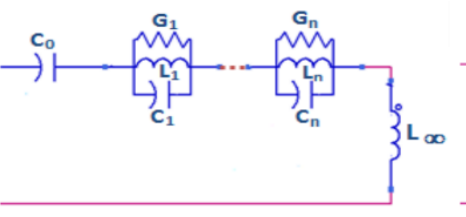

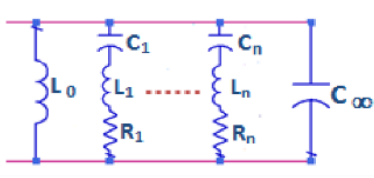

(b)
Fig. 16. Foster canonical forms of the equivalent circuit for (a) electric antennas and (b) magnetic antennas [19]

The input impedance $Z_{\text {in }}(\omega)$ of the antenna is modeled here using the equivalent network shown in Fig. 16:

$$
Z_{\text {in }}(\omega)=j \omega L_{0}+\frac{1}{j \omega C_{0}}+\sum_{n=1}^{N_{\max }} \frac{R_{n}}{1+j Q_{n}\left(\frac{\omega}{\omega_{n}}-\frac{\omega_{n}}{\omega}\right)}
$$

where $\omega_{n}=\left(L_{n} C_{n}\right)^{-0.5}$ and $Q_{n}=\omega_{n} R_{n} C_{n}$, $\omega$ is the operating radian frequency, $\omega_{\mathrm{n}}$ is the radian frequency of the $n$th resonant mode, $\mathrm{C}_{0}$ is the quasi-static input capacitance, $\mathrm{L}_{0}$ is an inductance that takes into account the higher order modes as well as for feeding effects, $\mathrm{N}_{\max }$ is the number of modes required to properly describe the frequency response of the antenna input impedance, while $C_{n}, L_{n}, R_{n}$ and $Q_{n}$ are the capacitance, inductance, resistance, and quality factor, respectively, describing the lumped resonance processes that take place in the antenna structure [19].

In the first step, the corresponding equivalent circuit model of the CMTL-loaded monopole antenna was chosen in this investigation. The details of the antenna design are shown in Fig. 17(a). The antenna layout with a total footprint of $30 \times 52.6 \times 1 \mathrm{~mm} 3$ was simulated on the commonly FR4 substrate with dielectric constant of $\varepsilon r=4.3$ and loss tangent of $\tan \delta=0.025$. The return loss result of the antenna is shown in Fig. 17(b). 


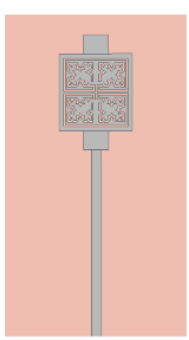

(a)

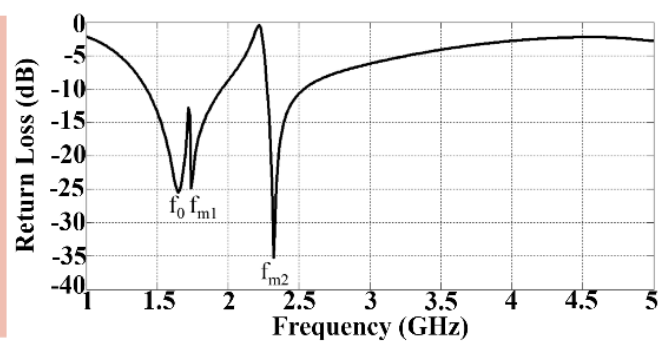

(b)
Fig. 17. (a) Layout of the CMTL-loaded monopole antenna (b) Simulated return loss of the CMTL-loaded monopole antenna.

The phase induced from the host monopole and the loaded CMTL element play a dominant role in determining the resonance frequency of antenna. The length of the monopole was designed to enable the antenna to operate around $1.65 \mathrm{GHz}\left(\mathrm{f}_{0}\right)$. But The CMTL loading leads to the slightly reduced operating frequency of the host monopole due to the coupling of the CMTL to the host monopole [13]. In addition, two reflection dips $\left(\mathrm{f}_{\mathrm{m} 1}=1.74 \mathrm{GHz}\right.$ and $\mathrm{f}_{\mathrm{m} 2}=2.32$ $\mathrm{GHz}$ ) with magnitude better than $-10 \mathrm{~dB}$ are achieved when CMTL elements are loaded in the monopoles. In the initial equivalent circuit model, as shown in Fig. 18, series inductance models the inductive effect of the signal strip, the first RLC branch models the host monopole Resonance frequency $\left(\mathrm{f}_{0}\right)$, the other two RLC branches model the impinging of the time-varying axial electric field to the outer CSSRRP and inner complementary Koch slot ( $\mathrm{f}_{\mathrm{m} 1}$ and $\mathrm{f}_{\mathrm{m} 2}$ ), respectively.

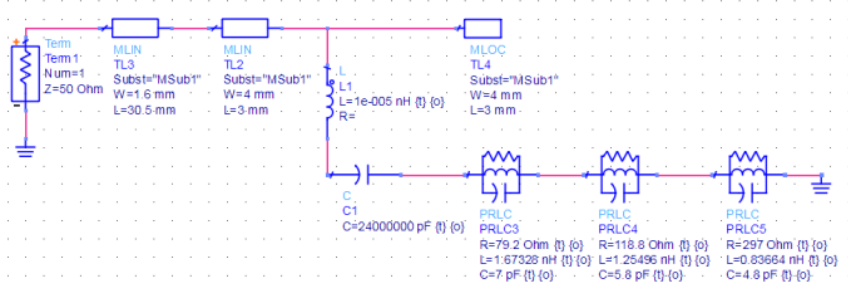

Fig. 18. Equivalent circuit model of the CMTL-loaded monopole antenna.

As shown in Fig. 18, $\mathrm{TL}_{3}, \mathrm{TL}_{2}$ and $\mathrm{TL}_{4}$ model the microstrip feed, matching, open-circuit microstrip sections of the antenna, respectively. Also, the inductance, capacitance and three series RLC branches model the radiation patch of the antenna. First the initial values of the parameters were calculated, then in order to get a more accurate model, an optimization algorithm was applied to obtain accurate values. This was done by minimizing an objective function expressed as follows [22]:

$$
H=\frac{1}{m} \sum_{k=1}^{m}\left[\frac{\left|s_{i j}^{T}\left(f_{k}\right)\right|-\left|s_{i j}^{E}\left(f_{k}\right)\right|}{\left|s_{i j}^{T}\left(f_{k}\right)\right|}\right]^{2}
$$

where $i, j=1,2, m$ is a number of simulated/measured frequency (f) points, $S^{T}$ ij are the S-parameters HFSS data, and $\mathrm{S}_{\mathrm{ij}}$ are the ADS simulated $\mathrm{S}$-parameters using the equivalent circuit values, which were obtained with the proposed method. A termination tolerance of $1 \times 10^{-10}$ was applied [22]. The optimization algorithm has been implemented in ADS by Optimization and Goal blocks, as shown in Fig. 19(b). The final values of the equivalent circuit elements obtained from optimization are shown in Fig. 18. Figure 20 compares the variation of the reflection coefficient responses with frequency as obtained from HFSS and ADS. It can be seen from the figure that there is a good agreement between the results of HFSS and ADS at the frequencies indicated in the Fig. 17(b) (the points marked $\mathrm{f}_{0}$, $\left.\mathrm{f}_{\mathrm{m} 1}, \mathrm{f}_{\mathrm{m} 2}\right)$. (a)

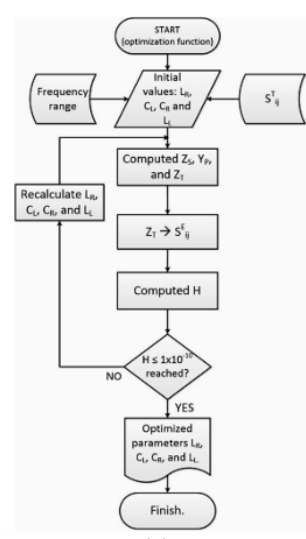

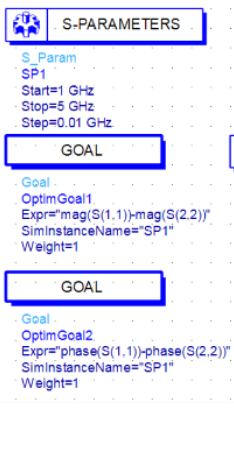

(b)
Fig. 19. (a) Optimization algorithm [22] (b) implementation of the optimization algorithm in ADS simulation.

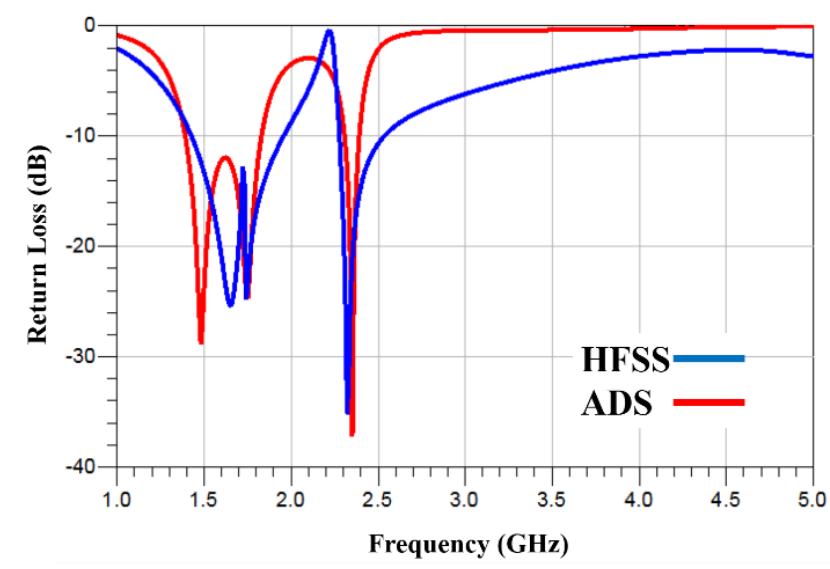

Fig. 20. Comparison of the reflection coefficient responses of the CMTLloaded monopole antenna obtained from HFSS and ADS.

In the second step, the equivalent circuit model of Antenna 1 was investigated. The proposed electrical model consists of two CMTL-loaded monopole equivalent circuit (Same as Fig. 18) that represented the equivalent circuit of each array antenna branch. The two equivalent circuits were connected by a single capacitance due to the coupling between the two radiation patches. The coupling capacitance was derived from the odd mode capacitance Bahl, Fig. 21 [20].

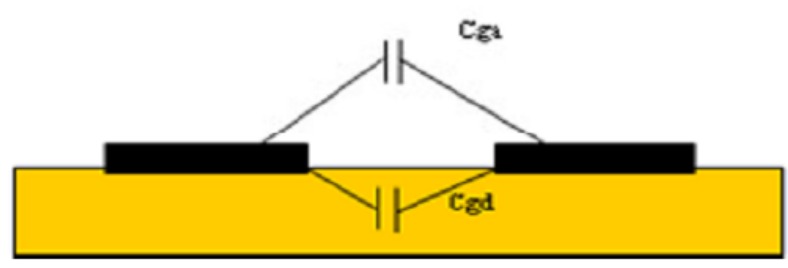

Fig. 21. Coupling capacitance of the two patch [20].

It can be calculated using the equation as follows [20]:

$$
C_{c}=C_{g a}+C_{g d}
$$




$$
\begin{gathered}
C_{g a}=\left\{\begin{array}{c}
\frac{\varepsilon_{0}}{\pi} \ln \left\{2 \frac{1+\sqrt{k^{\prime}}}{1-\sqrt{k^{\prime}}}\right\} 0 \leq k^{2} \leq 0.5 \\
\frac{\pi \varepsilon_{O}}{\ln \left\{2 \frac{1+\sqrt{k}}{1-\sqrt{k}}\right\}} 0.5 \leq k^{2} \leq 1
\end{array}\right. \\
k=\frac{d}{d+2 W}, k^{\prime}=\sqrt{1-k^{2}} \\
C_{g d}=\frac{\varepsilon_{0} \varepsilon_{r}}{\pi} \ln \operatorname{coth}\left(\frac{\pi d}{4 h}\right)+0.65 C_{f}\left\{\frac{0.02}{d / h} \sqrt{\varepsilon_{r}}+\left(1-\frac{1}{\varepsilon_{r}^{2}}\right)\right\}
\end{gathered}
$$

where $\mathrm{C}_{\mathrm{c}}$ is coupling capacitance of the two patch, $\mathrm{C}_{\mathrm{ga}}$ describes the gap capacitance in the air, $\mathrm{C}_{\mathrm{gd}}$ Represents the capacitance value due to the electric flux in the dielectric region, $\mathrm{W}$ is the width of the patch and $\mathrm{h}$ is the thickness of the dielectric substrate [20]. Basing on the equations developed in the previous paragraph, the coupling capacitance of Antenna 1 was calculated. Therefore, the equivalent model of Antenna 1 was obtained with respect to the coupling capacitor (With the red circle shown in the model), as shown in Fig. 22. Also, like the previous presented model, this model used the optimization algorithm in order to obtain more accurate values. Fig. 23 shows Comparison of the reflection coefficient responses of Antenna 1 obtained from HFSS and ADS. It can be seen that $\mathrm{S} 11$ are similar which prove that our model is accurate.

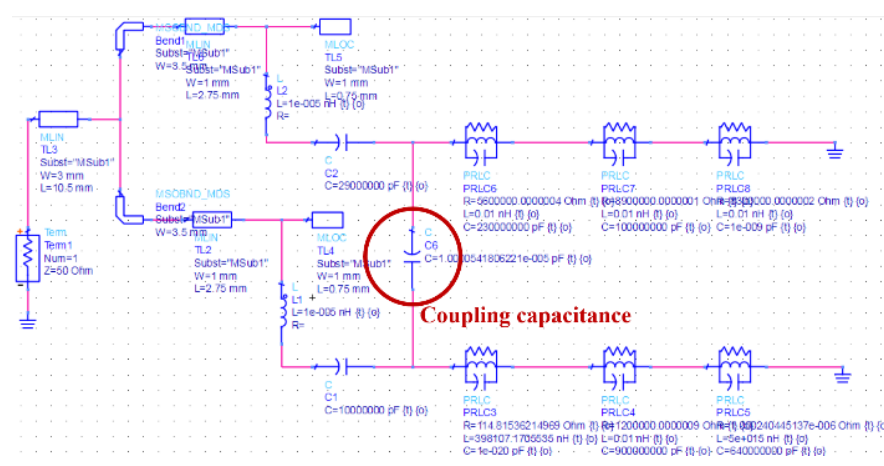

Fig. 22. Equivalent model of Antenna 1 in ADS

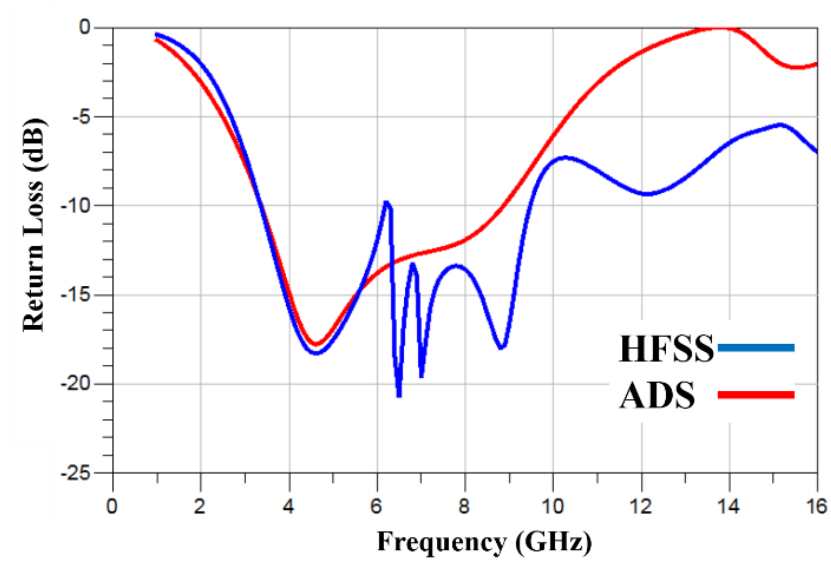

Fig. 23. Comparison of the reflection coefficient responses of Antenna 1 obtained from HFSS and ADS.

In the last step, the equivalent circuit model of Antennas $2 \& 3$ were investigated. In this case the electrical model can be constructed using the same technique. First we determined the equivalent model of each array branch using the obtained equivalent model of the CMTL-loaded monopole antenna (same as Fig. 18). Then we considered the coupling capacitors between the different radiation elements. Each capacitor represents the mutual coupling between each pair of radiation elements (With the red circles shown in the model). At last, using optimization, accurate values were obtained for the model parameters. The proposed model of Antennas 2 and 3 are shown in Fig. 24 and 25, respectively. As shown in the Figures, due to the simpler geometry of the Fibonacci tree, the equivalent model of Antenna 3 is simpler than Antenna 2, also it has less coupling capacitors. After building the electrical model, the ADS simulation results compared to those given by HFSS, as shown in Fig. 26. As can be seen, S11 are in good agreement which means that our model is very accurate. Besides, the simulation time of equivalent model by ADS is very shorter than the proposed array by HFSS simulator.

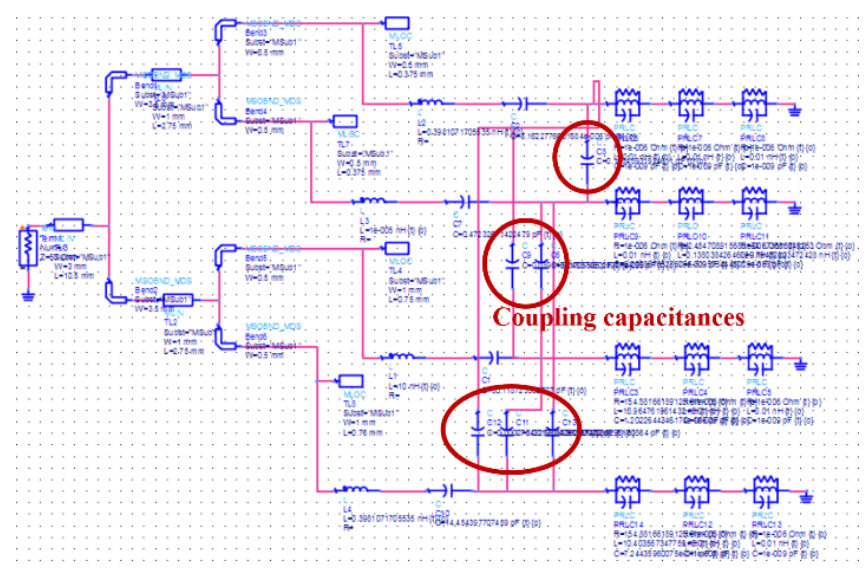

Fig. 24. Equivalent model of Antenna 2 in ADS.

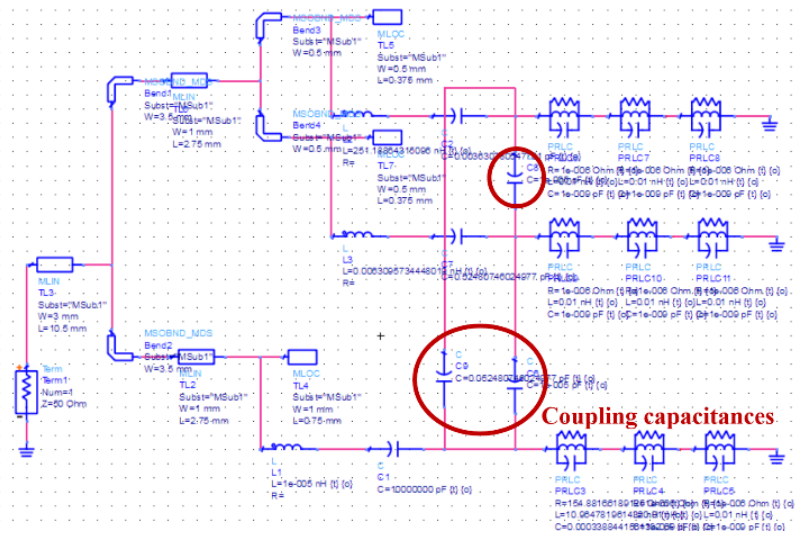

Fig. 25. Equivalent model of Antenna 3 in ADS. 


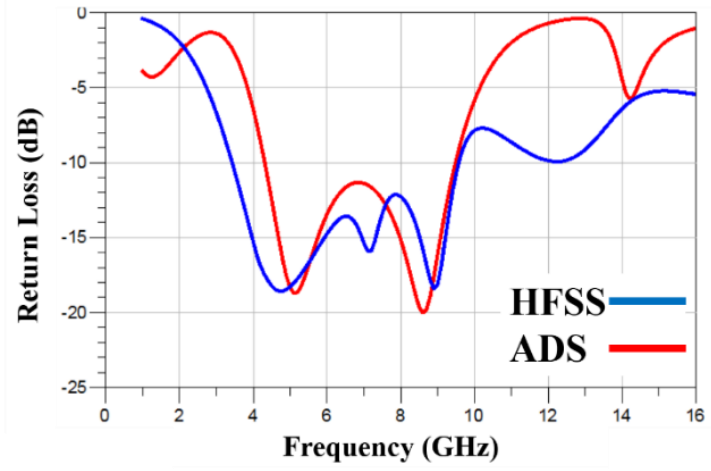

(a)

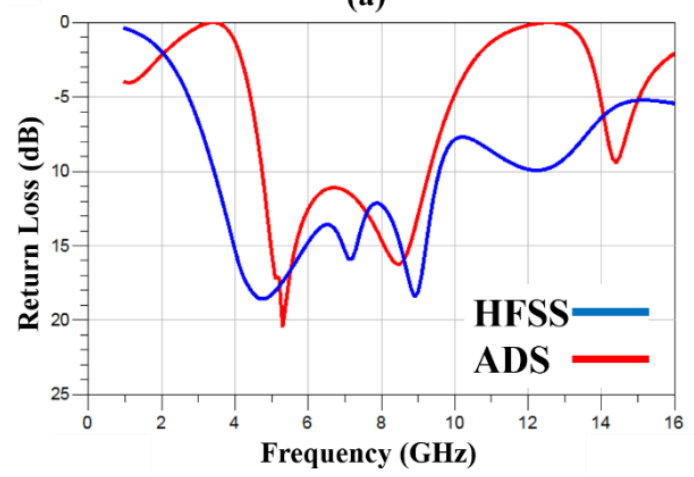

(b)

Fig.26. Comparison of the reflection coefficient responses obtained from HFSS and ADS: (a) Antenna 2 (b) Antenna 3.

\section{CONCLUSION}

A new family of the UWB MTM-loaded microstrip array antenna is proposed. The proposed antenna design consists of CMTL-loaded monopoles. The distributed CMTL elements, contain a Koch-shaped extended complementary single split ring resonator pair (K-ECSSRRP) etched on the signal strip. The left-handedness property of CMTL element is demonstrated by permeability and permittivity curves. Fibonacci and fractal geometric patterns utilized to design a new topology of the MTM-loaded array antennas. Simulation results confirm that the frequency bandwidth of the proposed antennas are increased and the RCS of them are decreased because of the metal area reduction. Then, this study presented an improved method to extract the parameters of an equivalent circuit model of the proposed array antenna. First, the equivalent model of the CMTLloaded monopole obtained in ADS based on Foster canonical form. Then, this study described the equivalent model of the coupling between array radiation elements in the case of fractal \& Fibonacci array antennas. The model responses using ADS showed good agreement with those obtained from the HFSS simulation. The proposed approach can be easily implemented without complicated mathematical programming methods. The proposed antenna represents omnidirectional radiation characteristics in the $\mathrm{H}$ plane for the frequency bands of interest, as requirement for the wireless applications. Also, the proposed antenna is intended for the use in wireless applications because of low profile, low cost, compactness, wide bandwidth and Low RCS.

\section{REFERENCES}

[1] R. Kumar Saraswat, and M. Kumar, "Miniaturized slotted ground UWB antenna loaded with metamaterial for WLAN and WiMAX applications," Progress in Electromagnetics Research B, vol. 65, pp. 65-80, 2016.

[2] Z. Mansouri, A. S. Arezomand, S. Heydari, and F. B. Zarrabi, "Dual notch UWB Fork monopole antenna with CRLH metamaterial load," Progress in Electromagnetics Research, Vol. 65, pp. 111-119, 2016.

[3] M. A. W. Nordin, M. T. Islam, and N. Misran, "Design of a compact ultrawideband metamaterial antenna based on the modified split-ring resonator and capacitively loaded strips unit cell," Progress in Electromagnetics Research, Vol. 136, pp. 157-173, 2013.

[4] E. Hanae, N. A. Touhami, and M. Aghoutane, "Miniaturized microstrip patch antenna with spiral defected microstrip structure," Progress In Electromagnetics Research Letters, Vol. 53, pp. 37-44, 2015.

[5] M. Rahimi, F. B. Zarrabi, R. Ahmadian, Z. Mansouri, and A. Keshtkar, "Miniaturization of antenna for wireless application with difference metamaterial structures," Progress In Electromagnetics Research, Vol. 145, pp. 19-29, 2014.

[6] R.K. Saraswat, and M. Kumar, "A frequency band reconfigurable UWB antenna for high gain applications," Progress In Electromagnetics Research B, Vol. 64, pp. 29-45, 2015.

[7] H. X. Xu, G. M. Wang, Y. Y. Lv, M. Q. Qi, X. Gao, and S. Ge "Multifrequency monopole antennas by loading metamaterian transmission lines with dual-shunt branch circuit," Progress In Electromagnetics Research, Vol. 137, pp. 703-725, 2013.

[8] S. Nelaturi, N. V. S. N. Sarma, "A Compact Microstrip Patch Antenna Based on Metamaterials for Wi-Fi and WiMAX Applications," Journal of Electromagnetic Engineering and Science, vol. 18, no. 3, pp. 182-187, 2018.

[9] S. N. Boyko, A. S. Kukharenko, and Y. S. Yaskin, "EBG Metamaterial Ground Plane for Mitigation of Multipath Signals in GNSS Antenna," Journal of Electromagnetic Engineering and Science, vol. 15, no. 4, pp. 199-205, 2015.

[10] R. Datta, T. Shaw, and D. Mitra, "Miniaturization of microstrip Yagi array antenna using metamaterial," Progress in Electromagnetics Research, Vol.72, pp. 151-158, 2017.

[11] X. W. Dai, Z. Y. Wong, L. Li, and C. H. Liong, "Multi-band rectangular microstrip Antenna using a metamaterial-inspired technique," Progress in Electromagnetics Research Letters, Vol.41, pp. 87-95, 2013.

[12] H. H. Yang, X. Y. CAO, Q. R. Zheng, J. J. MA, and W. Q. Li, "Broadband RCS reduction of microstrip patch antenna using bandstop frequency selective surface," Radioengineering, Vol.22, NO.4, pp.1275-1280, 2013.

[13] H. X. Xu, G. M. Wang, M. Q. Qi, C. X. Zhang, J. G. Liang, J. Q. Gong, and Y. C. Zhou, "Analysis and design of two-dimensional resonant-type composite right/left-handed transmission lines with compact gain-enhanced resonant antennas," IEEE Transactions on Antennas and Propagation, vol. 61, no. 2, pp. 735-747, 2013.

[14] B. Ozbakis, and A. Kustepeli, "The resonant behavior of the Fibonacci fractal tree antennas," Microwave and optical technology letters, Vol. 50, no.4, pp. 1046-1050, 2008.

[15] S. Khobragade, S. Nalbalwar, and A. Nandgaonkar, "Study of fractal tree antenna for multiband applications," in Proceedings of the International Conference on Communication and Signal Processing (ICCASP), Lonere, India, 2016, pp. 522-529.

[16] S. Shrestha, S. R. Lee, and D. Y. Choi, "A new fractal-based miniaturized dual band patch antenna for RF energy harvesting," International Journal of Antennas and Propagation, Vol. 2014, article no. 805052,2014

[17] R. kubacki, S. Lamari, M. Czyzewski, and D. Laskowski, "A broadband left-handed metamaterial microstrip antenna with doublefractal layers," International Journal of Antennas and Propagation, vol. 2017, article no. 6145865, 2017.

[18] C. Sharma and D. K. Vishwakarma, "Miniaturization of Spiral Antenna Based on Fibonacci Sequence Using Modified Koch Curve," in IEEE Antennas and Wireless Propagation Letters, vol. 16, pp. 932935, 2017

[19] K. H. Sayidmarie, and L. S. Yahya, "Modeling of Dual-Band Crescent-Shape Monopole Antenna for WLAN Applications," International Journal of Electromagnetics and Applications, 2014.

[20] A. Ferchichi, N. Fadlallah, and A. Gharssallah, "A novel electrical model to an antenna array," Journal of Engineering and Technology Research, Vol. 3, no. 12, pp. 321-329, 2011.

[21] A. B. Numan, and M. S. Sharawi, "Extraction of Material Parameters for Metamaterials Using a Full-Wave Simulator," IEEE Antennas and Propagation Magazine, Vol. 55, no. 5, pp. 202-211, 2013. 
[22] E. O. Ezenwa, M.C. Maya-Sanchez, and J. A. R. Hernandez, "Improved method for extracting the equivalent circuit elements of a CRLH-TL unit cell," Revista Mexicana de Fisica, 2015.

[23] B. R. Shookooh, A. Monajati, and H. Khodabakhshi, "The novel design and modeling of Ultra-Wideband metamaterial-loaded microstrip array antenna using the fractal \& Fibonacci geometric patterns," Journal of Electromagnetic Engineering and Science, vol. 20, no. 1, pp. 53 63, 2020 .

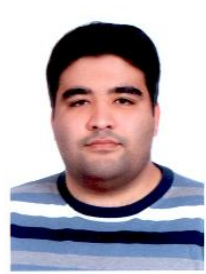

Besharat Rezaei Shookooh was born in Tehran, Iran 1987. He is Ph.D. student in Electrical Engineering at Islamic Azad University, Yadegar-e-Imam Khomeini (RAH) Shahre Rey Branch. From 2014 until now, he is a Teacher Assistant with the Department of Electrical Engineering, Islamic Azad University, Shahre-Rey Branch, Iran. His research interests include analysis and design of microstrip antennas, design and modeling of microwave structures, optic and electromagnetic theory.

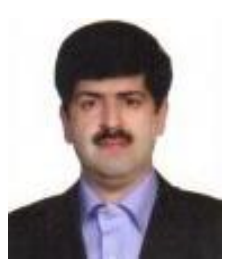

Alireza Monajati was born in Tehran, Iran 1970. He received his Ph.D degree in Electrical Engineering from Azad University, Scineces and researchers. From 1997 until now, he is a Teaching Assistant with the Department of Electrical Engineering, Islamic Azad University, Shahre-Rey Branch, Iran. His research interests include analysis and design of microstrip antennas, design and modeling of microwave structures, optic and electromagnetic theory.

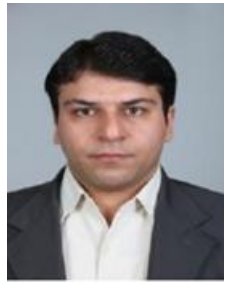

Hamid Khodabakhshi was born in Iran, in 1979. He received the B.S. and M.S. degrees in electrical engineering from the University of Tehran (UT), Tehran, Iran, in 2000 and 2002, respectively. He gained the $\mathrm{Ph} . \mathrm{D}$. degree in electrical engineering from the Iran University of Science and Technology (IUST), Tehran, Iran in 2011. His research activities and interests include microstrip antennas, electromagnetic compatibility (EMC)/ electromagnetic interference (EMI), reconfigurable antenna, 5G antennas and biological effects of EMfields. 